\title{
Effects of bending on a flexible metamaterial absorber
}

\author{
Siti Nurzulaihan Isa ${ }^{1}$, Osman Ayop ${ }^{2}$, Abu Sahmah Mohd Supa'at ${ }^{3}$, Mohammad Kamal A. Rahim ${ }^{4}$, \\ Noor Asniza Murad ${ }^{5}$, Farid Zubir ${ }^{6}$, Huda A. Majid ${ }^{7}$ \\ ${ }^{1,2,3,4,5,6}$ School of Electrical Engineering, Faculty of Enginering, Universiti Teknologi Malaysia, Malaysia \\ ${ }^{7}$ Faculty of Engineering Technology, Universiti Tun Hussein Onn Malaysia, Malaysia
}

\begin{tabular}{|c|c|}
\hline Article Info & ABSTRACT \\
\hline Article history: & This paper presents a study of bending a metamaterial based absorber. \\
\hline & The study of bending is important for textile material since it can be easily \\
\hline Recelved Dec 4, 2019 & crumpled. The basic absorber that is simulated for the study is an annulled \\
\hline Revised May 18, 2020 & circle as the top patch, and metal ground plane that sandwich a textile-based \\
\hline Accepted Jun 22, 2020 & $\begin{array}{l}\text { substrate. The center frequency for the absorber is } 10.525 \mathrm{GHz} \text {. The type } \\
\text { of bending is divided into two parts, which is convex bending and concave }\end{array}$ \\
\hline Keywords: & $\begin{array}{l}\text { bending. Through series of simulations, the effects of the bending on } \\
\text { the absorptivity and the shifting of the resonant frequency is observed. }\end{array}$ \\
\hline $\begin{array}{l}\text { Bending effect } \\
\text { Concave convex } \\
\text { Flexible material }\end{array}$ & $\begin{array}{l}\text { Also, the study on the change of incident and polarization angle is also } \\
\text { included to support the basis of flexible metamaterial absorber affected by } \\
\text { the bending. }\end{array}$ \\
\hline
\end{tabular}

This is an open access article under the CC BY-SA license.

Metamaterial absorber

Stealth technology

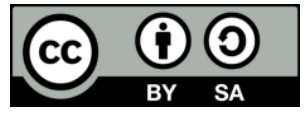

Corresponding Author:

Osman Ayop,

School of Electrical Engineering,

Universiti Teknologi Malaysia, Malaysia.

Email: osman@fke.utm.my

\section{INTRODUCTION}

Ever since Landy et al. introduced perfect metamaterial absorber (MMAb), MMAb has become one of the most potential study in microwave research study [1]. Metamaterial is an artificially structured material that is capable to wield the electromagnetic radiation according to the purpose of its application. Structures that vary in size, shape and composition, inducing electromagnetic moments, that affect the macroscopic effective permittivity and permeability successively [2]. Absorber is one of the applications that can use the metamaterial as its material. The function of absorber is to absorb the electromagnetic radiation, in the same time requires a very minimal reflectance. As for MMAb, the metallic patch serves as admittance. High absorptivity can be realized when a very low reflectance occurred, which is resulted by a matching load impedance to the free space [3].

MMAb has been increasing in popularity among researchers due to its uniquess property that make use of lossy component (imaginary part) of effective permittivity and permeability of the designated structures rather than focusing to the real part of the aforementioned medium properties that are normally interest the researchers. A lot of devices can be designed using the concept of MMAb such as bolometer spectroscopy [4, 5], thermal emission [6,7], and sub-wavelength imaging [8,9]. Since metamaterial has infinite possibilities to design, it has attracted huge attention to diversify its field. To date there are various MMAb designed, such as single-band [10, 11], dual-band [12, 13], multi-band [14, 15], wide-band [16, 17], tunable or switching [18, 19], and flexible MMAb [20]. The flexible MMAb is a challenge to be realized as to match the load impedance with the free space at various inclination is not easy. The first step to do other than varying its incident angle is to choose the correct material for flexibility of MMAb. Many designs of 
the MMAb are based from FR-4, which is not only economically feasible, but its light and thin structure make it possible to create a good MMAb. However, it is hard and not flexible, which makes it not suitable for the purpose. Secondly is to deform the structure without destroying its shape. The method proposed in this paper is by bending the MMAb in two ways; convex and concave bending, which will be later discussed in next section. The idea of this MMAb is to function against a motion detector; function-wise it will sound like an invisibility cloak. To create a MMAb that function against a motion detector means that it needs flexibility to operate against all incident wave, including some bending effects on no matter polarization it might be [21]. The most suitable material to operate on is textile-based material. Since the MMAb designed to be operated in X-Band, a unit cell is predicted to be very small and inapplicable to textile, thus it limits the posture of the MMAb [22]. Therefore, for this paper only two types of bending are introduced.

\section{DESIGN CONSIDERATION}

\subsection{Metamaterial absorber}

To achieve the purpose of stealthy against electromagnetic radiation, the MMAb is aimed to be light and flexible. The best materials for the task is textile-based for substrate and a very thin metallic plate for the patch and the ground plane. The textile substrate is projected to be $1.0 \mathrm{~mm}$ thin and dielectric constant, $\varepsilon_{r}$ of 1.7 with tangent loss of 0.02 , operating at $2.45 \mathrm{GHz}$ [23]. The closest material would be denim. The copper plate has an electric conductivity of $5.8 \times 10^{7} \mathrm{~S} / \mathrm{m}$ with the thickness of $0.035 \mathrm{~mm}$. The rational of choosing the thinnest material possible is based on the study that the greater absorptivity can be achieved through thinner absorber [24]. The structure chosen for analysis is in the form of conventional ring structure as shown in Figure 1 due to its simple design and high absorbance [25]. Figure 1 is the design of metamaterial absorber used for the simulation of bending absorber. The selection of this design is based on the orthodox structure and as to achieve the polarization insensitive purpose of flexible metamaterial absorber, the design has to be symmetrical at the front view. The resonant frequency of this structure is $10.525 \mathrm{GHz}$.

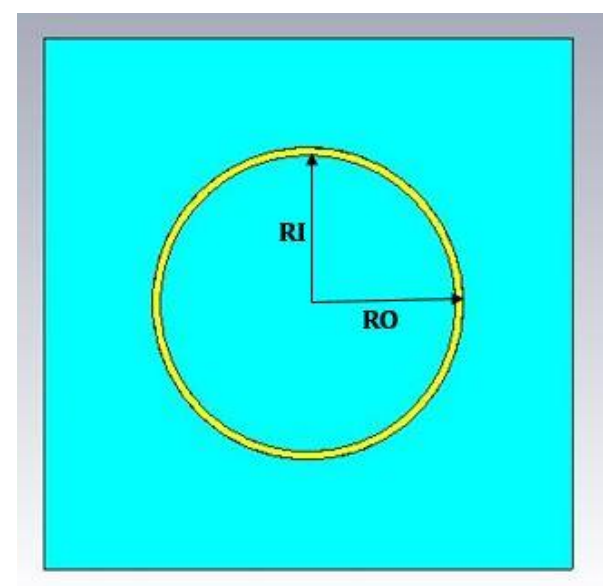

Figure 1. Effects of selecting different switching under dynamic condition

\subsection{Types of bending}

The curvature set for the bending factor is decided on the relation between radius of the curvature and the width of the unit cell, as shown in Figure 2(a). As the width of a unit cell is labelled as $W$, the radius, $R$ is initially a part of a cylinder, at which the absorber bent accordingly to the curvature. Table 1 explains the difference in radius that makes up the bending factor, which is the manipulating variable of the simulation. The bending factor is prescribed as the larger the value, the less significant the bending will be, and zero-bending factor means that there is no bending happened. Bending factor of 0.3 is the limit for the simulation as it is difficult to extract the result by using smaller bending factor. There are two parts of the bending factors, one is curvature that bends towards the electromagnetic radiation, or Port 1 , which is called concave curvature and another bending factors whose curvature bends away from the radiation that is called convex curvature. The posture of both concave and convex curvature are pictured in Figure 2(b) and Figure 2(c). 


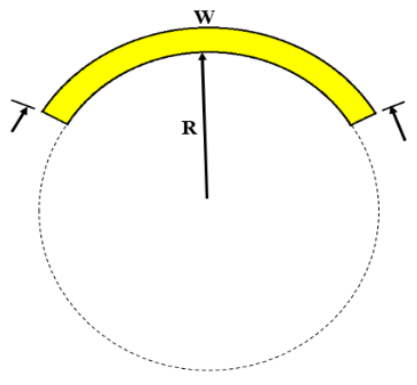

(a)

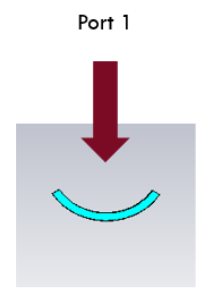

Port 2

(b)

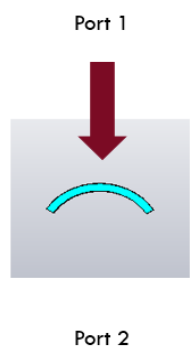

(c)

Figure 2. Bending, (a) The curvature set for the bending factor of selecting different switching under dynamic condition, (b) Concave bending, (c) Convex bending

Table 1. Setting of bending factor

\begin{tabular}{ccc}
\hline Curvature & \multicolumn{2}{c}{ Bending factor } \\
\cline { 2 - 3 } radius, $\mathrm{R}$ & Concave bending & Convex bending \\
\hline $5 / 3 * \mathrm{~W}$ & 1.7 & 1.7 \\
$3 / 2 * \mathrm{~W}$ & 1.5 & 1.5 \\
$5 / 4 * \mathrm{~W}$ & 1.3 & 1.3 \\
$\mathrm{~W}$ & 1.0 & 1.0 \\
$3 / 4 * \mathrm{~W}$ & 0.7 & 0.7 \\
$1 / 2 * \mathrm{~W}$ & 0.5 & 0.5 \\
$1 / 3 * \mathrm{~W}$ & 0.3 & 0.3 \\
\hline
\end{tabular}

\section{PARAMETRIC STUDY}

There are three series of simulations; first to find out how the bending factor affects the absorptivity, second is to differ in term of incident angle and the third is to look the effects change of the polarization angle.

\subsection{Absorptivity for different bending factor}

The effects of bending of the MMAb in convex and concave manner can be shown in Figure 3(a) and Figure 3(b) respectively. The initial hypothesis of MMAb is, the more deformed a MMAb is, the worse the absorptivity it will achieve. As explained in Table 1, the more obvious the bending happened to the MMAb, the smaller it is for the bending factor. Referring to Figure 3(a), the flat annulled circle MMAb, the one with no bending applied, reaches the maximum absorptivity at $10.525 \mathrm{GHz}$, which is around $90 \%$, and the absorbance graph shifted to the right as the bending factor decreases, for convex bending. However, for concave bending, the absorbance graph shifted to the left as the bending becomes more obvious. The smallest bending factor, 0.3 , records the worst absorptivity of all at its center frequency, which is $81.42 \%$ at $10.61 \mathrm{GHz}$. However, it gained $71.55 \%$ of absorptivity at $10.525 \mathrm{GHz}$, which surpasses bending factor of 0.5 that reached the lowest of $66.38 \%$ at the same frequency. The overall finding from convex bending is that, the increase or decrease of bending factor does not improve the absorptivity of the MMAb.

As for concave bending as shown in Figure 3(b), the MMAb shows a more consistent absorptivity as the bending occurs. The peak absorptivity of each run for different bending factors is slightly better than the one run at normal inclination. Similar behavior with convex bending, as the bending factor gets smaller, the absorptivity deteriorates at the designated center frequency. The worst absorptivity recorded is $61.45 \%$ for bending factor 0.3. Overall, absorptivity of annulled circle MMAb when bending is applied, does not show a fair distribution behavior and it is hardly predictable. However, it is proven that the more flexible the MMAb is, the lower the absorptivity it may achieve, with exception of bending factor 0.5 for convex bending. Comparing both bending, concave bending exhibits more stable absorptivity across different bending factors at $10.525 \mathrm{GHz}$, although it has worse absorbance when applying lowest bending factor.

Figure 4 shows the absorptivity for both convex and concave bending over the change of bending factor. As assumed earlier, the larger the bending factor is, the greater absorptivity it can achieve. The assumption is true for both cases, however, for concave bending it shows a sudden drop at bending factor of 0.5 and 1.3. The absorptivity pattern is more stable for concave bending, although it illustrates a relatively worse absorbance at bending factor lower than 0.5. At average, convex bending scores higher in term of absorptivity. On the other hand, concave bending exhibits high absorptivity of $72.16 \%$ at bending factor of 0.3 . 


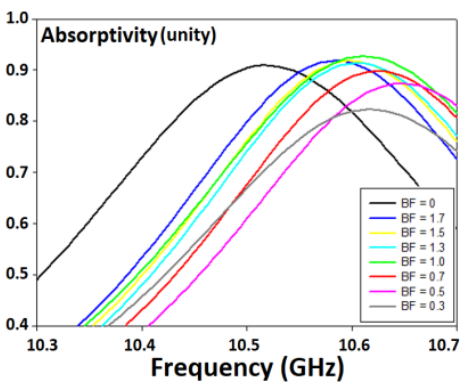

(a)

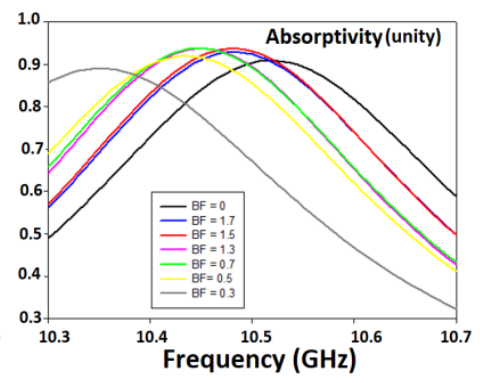

(b)

Figure 3. Absorptivity of different bending factor for, (a) Convex bending, (b) Concave bending

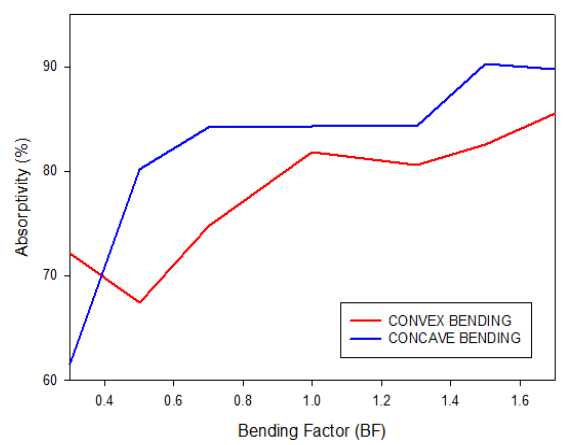

Figure 4. Absorptivity over changing bending factor for convex bending and concave bending

\subsection{Varying the incident angle}

This section explains the absorptivity pattern for changing bending factor. Referring to Figure 5(a), the trend of absorptivity of all incident angles for convex bending is similar. For every inclination, the absorptivity reaches the lowest at bending factor of 0.5 . The divergence for the largest incident angle is only $\pm 6 \%$, which is the lowest compared to other incident angles. This means that at larger incident angle for convex bending, the change of bending factor appears to affect the absorptivity less when compared to change of bending factor at smaller incident angle. However, the very low of absorptivity for incident angle of $70^{\circ}$ makes the MMAb unfit to be called flexible MMAb. It can be concluded from Figure 5(a) that the larger incident angle will deteriorate the absorptivity more.

Figure 5(b) illustrates the trend of absorptivity of different incident angles of concave bending. The pattern slightly differs, as when the MMAb is at flat inclination and at the largest bending factor, the absorptivity is not relatively high that is $89.77 \%$, compared to the absorptivity at $30^{\circ}$ and $50^{\circ}$, which are $93.72 \%$ and $94.36 \%$. At every incident angle, the absorptivity increases greatly after applying the bending factor of 0.5 . Three out of four simulated incident angles peak at bending factor 1.5 and it obviously shows when tilting the MMAb to $70^{\circ}$.

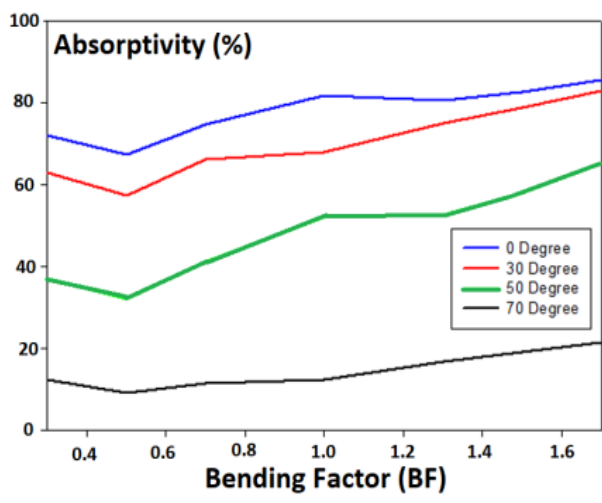

(a)

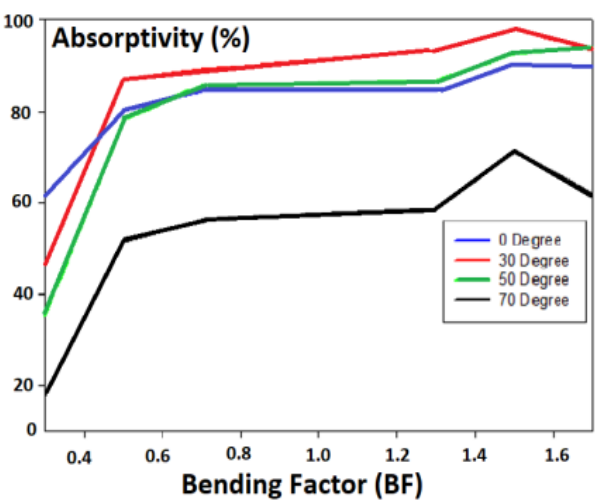

(b)

Figure 5. Absorptivity over bending factor for different incident angles, (a) Convex bending, (b) Concave bending

\subsection{Varying the polarization angle}

The absorptivity along the change of polarization angle is shown in Figure 6. As the design is horizontally and vertically symmetrical at top view of the MMAb, the simulation only took place from $0^{0}$ to $90^{\circ}$. The absorptivity achieved by the proposed MMAb fluctuates very slightly for both convex and concave bending. Although convex bending exhibits higher absorptivity, the difference is so small. The divergence of the absorptivity for the convex bending is $\pm 0.015 \%$ and for concave bending is around $\pm 0.01 \%$. Although various bending factor has been applied, the results are similar. From the observation, it can be assured that both of the MMAb designs are insensitive towards polarization as the deviation is insignificant. 


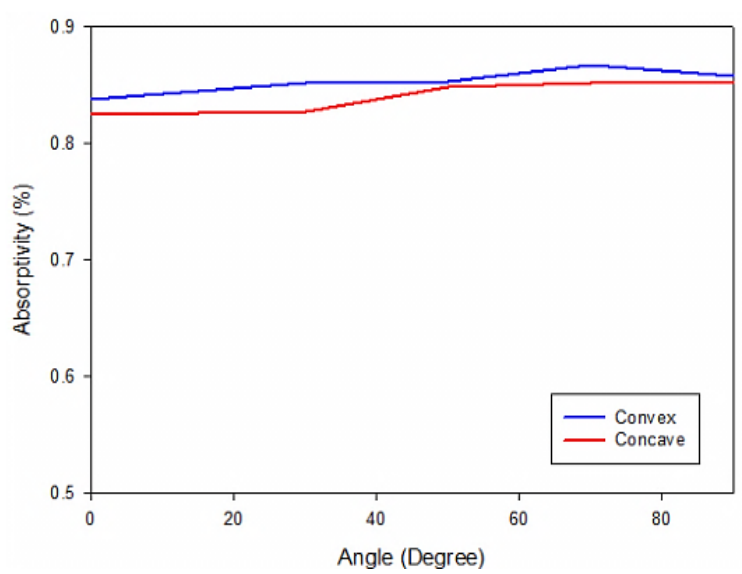

Figure 6. Absorptivity over polarization angle

\section{CONCLUSION}

Although the bending is only applied in simulation, how it will affect the absorptivity has been studied. It gives more opportunity to apply the theoretical part of the MMAb to practical. The assumption made prior to the study is proven true, that is the more deformed structure will generate less absorptivity. Additional result found is that, the center frequency changes as the bending takes place, either it is convex bending or concave bending. Based on existing work, it is possible to further the study to practical work and improve the structure, so that it is unaffected from every possible projection.

\section{ACKNOWLEDGEMENTS}

The authors thank the Ministry of Education (MOE) for supporting the research work and Research Management Centre (RMC), School of Postgraduate Studies (SPS) and School of Electrical Engineering (SKE), Faculty of Engineeering, Universiti Teknologi Malaysia (UTM), Johor Bahru, for the support of the research under Grant No. 04G69. The authors would also like to acknowledge all members of Advanced RF and Microwave Research Group (ARFMRG).

\section{REFERENCES}

[1] N. I. Landy, S. Sajuyigbe, J. J. Mock, D. R. Smith, W. J. Padilla, “A perfect metamaterial absorber”, Physical Review Letters, vol. 100, p. 207402, 2008.

[2] D. Lim, D. Lee, S. Lim “Angle- and polarization-insensitive metamaterial absorber using via array," Scientific Reports, vol. 6,no. 39686, 2016.

[3] C. A. Balanis, "Antenna Theory: Analysis and Design," 3ed, Wiley Interscience, 2005.

[4] K. B. Alici, "Hybridization of Fano and Vibrational Resonances in Surface-Enhanced Infrared Absorption Spectroscopy of Streptavidin Monolayers on Metamaterial Substrates," in IEEE Transactions on Nanotechnology, vol. 13, no. 2, pp. 216-221, 2014, March.

[5] S. Wolf, U. Strom, J. Culbertson and D. Paget, "Superconducting granular NbN bolometer for ultrafast spectroscopy," in IEEE Transactions on Magnetics, vol. 21, no. 2, pp. 920-923, March 1985.

[6] Q. Liu, H. Huang, W. Qin, K. Fu and X. Li, "An Extended 3-D Radiosity-Graphics Combined Model for Studying Thermal-Emission Directionality of Crop Canopy," in IEEE Transactions on Geoscience and Remote Sensing, vol. 45, no. 9, pp. 2900-2918, Sept. 2007.

[7] Jindi Wang, Xiamen Li, Hongbo Su and Ziti Jiao, "Approach on directional thermal emission modeling for 3-D structural pixel and the model validation," IGARSS 2001. Scanning the Present and Resolving the Future. Proceedings. IEEE 2001 International Geoscience and Remote Sensing Symposium (Cat. No.01CH37217), Sydney, NSW, Australia, vol.4, pp. 1985-1987, 2001.

[8] M. Ghosh and S. Kar, "Metamaterial plane-slab focusing and sub-wavelength imaging-The concept, analysis and characterization," 2013 Science and Information Conference, London, pp. 670-674, 2013.

[9] Yan Chen, Xiaohua Teng and Yijun Feng, "Loss Effect on Sub-wavelength Imaging by Compensated Bilayer of Anisotropic Metamaterials," 2006 IEEE Antennas and Propagation Society International Symposium, Albuquerque, NM, pp. 3361-3364, 2006.

[10] MM Gajibo, MKA Rahim, NA Murad, O Ayop, BD Bala, HA Majid, "X-band operations metamaterial absorber with extended circular ring topology for size reduction," Indonesian Journal of Electrical Engineering and Computer Science, vol. 6, no. 1, pp. 180-184, April 2017. 
[11] J. Wang et al., "Three dimensional microwave metamaterials absorbers composed of coplanar electric and magnetic resonators," Progress in Electromagnetics Research Letters, vol. 7, pp. 15-24, 2009.

[12] J. Song, L. Wang, M. Li, and J. Dong, "A dual-band metamaterial absorber with adjacent absorption peaks," Journal of Physics D: Applied Physics, vol. 51, no. 38, p. 385105, 2018.

[13] A. Hoque, M. Tariqul Islam, A. F. Almutairi, T. Alam, M. Jit Singh, and N. Amin, "A Polarization Independent Quasi-TEM Metamaterial Absorber for X and Ku Band Sensing Applications,” Sensors, vol. 18, no. 12, p. 4209, 2018.

[14] O. Ayop, M. K. A. Rahim, N. Murad, N. A. Samsuri, and R. Dewan, "Triple Band Circular Ring-Shaped Metamaterial Absorber for X-Band Applications," Progress In Electromagnetics Research M, vol. 39, pp. 65-75, 2014.

[15] H. Li, L. Hua Yuan, B. Zhou, X. Peng Shen, Q. Cheng, and T. Jun Cui, "Ultrathin multiband gigahertz metamaterial absorbers," Journal of Applied Physics, vol. 110, pp. 014909-014909, 2011.

[16] W. Zuo, Y. Yang, X. He, C. Mao and T. Liu, "An Ultrawideband Miniaturized Metamaterial Absorber in the Ultrahigh-Frequency Range," in IEEE Antennas and Wireless Propagation Letters, vol. 16, pp. 928-931, 2017.

[17] O Ayop, MKA Rahim, NA Murad, NA Samsuri, "Wideband polarization-insensitive metamaterial absorber with perfect dual resonances," Applied Physics A, vol. 122, no. 4, p. 316, 2016.

[18] B. Ma, S. Liu, X.-K. Kong, Y. Jiang, J. Xu, and H. Yang, "A Novel Wide-band Tunable Metamaterial Absorber Based On Varactor Diode/Graphene," Optik - International Journal for Light and Electron Optics, vol. 127, no. 5, pp. 3039-3043, 2016.

[19] M. Yoo and S. Lim, "Switchable Electromagnetic Reflector/Absorber with Electric-Field-Coupled LC Resonator," Electromagnetics, vol. 34, no. 5, pp. 421-429, 2014.

[20] Siti Nurzulaiha Isa, Osman Ayop, Farid Zubir, Noor Asniza Murad, Mohammad Kamal A Rahim, "Floral structure for textile-based metamaterial absorber," Indonesian Journal of Electrical Engineering and Informatics IJEEI, vol. 7, 2, pp. 375-381, 2019.

[21] T. Wanghuang, W. Chen, Y. Huang, G. Wen, "Analysis of Metamaterial Absorber in Normal and Oblique Incidence by using Interference Theory," AIP Advances, vol. 3, no. 10, p. 102118, 2013.

[22] F. Capolino "Metamaterials Handbook," Taylor and Francis, 2009.

[23] S. Sankaralingam and B. Gupta, "Determination of Dielectric Constant of Fabric Materials and Their Use as Substrates for Design and Development of Antennas for Wearable Applications," in IEEE Transactions on Instrumentation and Measurement, vol. 59, no. 12, pp. 3122-3130, Dec. 2010.

[24] O. Ayop, M. K. A. Rahim and N. A. Murad, "Wide angle and polarization insensitive circular ring metamaterial absorber at $10 \mathrm{GHz}, " 2013$ Proceedings of the International Symposium on Antennas \& Propagation, Nanjing, pp. 1295-1297, 2013.

[25] O. Ayop, M. K. A. Rahim, N. A. Murad and N. A. Samsuri, "Dual resonance circular ring-shaped metamaterial absorber with wide operating angle," 2015 International Symposium on Antennas and Propagation (ISAP), Hobart, TAS, pp. 1-4, 2015.

\section{BIOGRAPHIES OF AUTHORS}

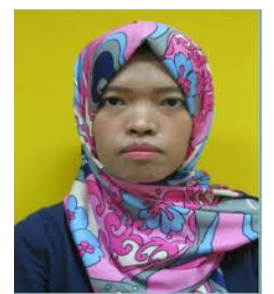

Siti Nurzulaiha Isa received her Master Degree in Electrical Engineering (Electronic \& Communication) from Universiti Teknologi Malaysia (2018), specialized in Telecommunication Engineering. As for Bachelor Degree, she graduated from University of Applied Sciences Berlin (Hochschule für Technik und Wirtschaft Berlin) in 2016 majoring in Telecommunication Engineering. She had experienced working as an intern in a radio wave and antenna company, Rohde \& Schwarz GmbH Co. \& KG, Berlin. During her post-graduate study, she had done her researches in microwave and antenna. Her title of thesis is X-band Matamaterial absorber for anti-motion detector shown a great contribution in the field of metamaterial absorber due to the great flexibility of the designed structures. She also was nominated as on of the excellent students graduated in 2018. Due to her great achievement in academic, she has ben offered a position as a procurement specialist cum purchaser in a specialty chemical company, Evonik Malaysia Sdn. Bhd.

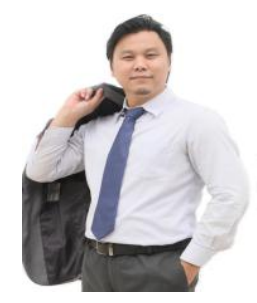

Osman Ayop received his Ph.D in Electrical Engineering (2016), M.Eng. (RF \& Microwave) (2010) and B.Eng. in Electrical majoring in Telecommunication (2007) degree from the Universiti Teknologi Malaysia (UTM). In 2013, He was also done his attachment in Uppsala University of Sweden under the supervision of Prof. Dr Vernon Cooray. He is currently serves as a Senior Lecturer in the Department of Communication Engineering, School of Electrical Engineering, UTM. His current research interest and specialization are in the field of antennas (Microstrip antenna, planar array antenna, ultra-wideband antenna and dielectric resonator antenna), propagation, and also metamaterials (electromagnetic band gap, left-handed, absorber, and artificial magnetic conductor). He has been published numerous of journals, proceedings, research books, and book chapters. He also won several university and national awards related to his research works. He has been appointed as a reviewer for several national/international journals and conferences. He is also an active researcher in Advanced RF and Antenna Research Group (ARFMRG) and has been participated in a lot of courses, seminars, exhibition etc. 

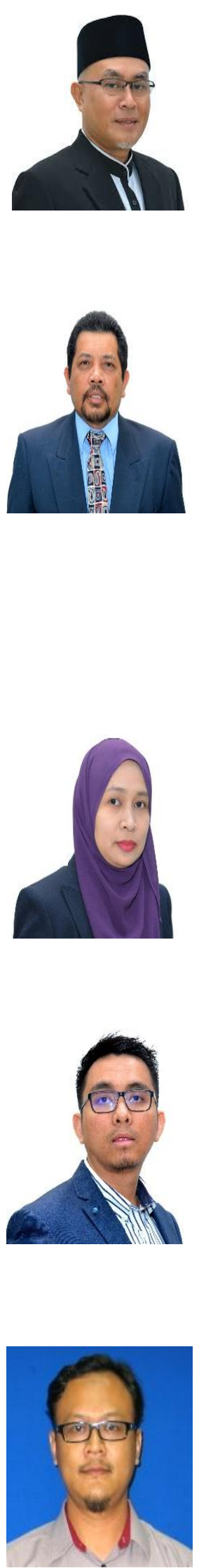

Abu Sahmah Mohd Supa'at was born in Johor Bahru, Malaysia on July 1963. He received his $\mathrm{PhD}$ degree from Universiti Teknologi Malaysia (UTM) in 2004. He joined Faculty of Electrical Engineering, UTM in 1986 as an Assistant Lecturer. He has taught various subjects and worked on a variety of research projects in the field of Optical Fibers, Free Space Optic, Visible Free Space Optic and PLC, and Photonic Devices. With the expertise gained in Photonics Switching Devices, he has been working as a visiting researcher in Mesa Research Institute, University of Twente the Netherland, ZenPhotonics Inc. and ChemOptics Inc. both are in Korea and a visiting professor in Pusan National University of Korea and City University of Hong Kong. To date he has written more than 100 papers, which has been published in National and International Journals as well as in the conferences. He is presently a Professor and the Deputy Dean (Development) Faculty of Electrical Engineering, Universiti Teknologi Malaysia.

Mohamad Kamal A Rahim received the B Eng. degree in Electrical and Electronic Engineering from University of Strathclyde, UK, in 1987. From 1987 to 1989, he worked as a Management Trainee at Sime Tyres Mergong Alor Star Kedah and Production Supervisor at Sime Shoes in Kulim Kedah. In 1989, he joined the Department of Communication Engineering, Faculty of Electrical Engineering Universiti Teknologi Malaysia Kuala Lumpur as an Assistant Lecturer A. He obtained his M.Eng Science from University of New South Wales Australia in 1992 and PhD degrees in Electrical Engineering from University of Birmingham UK in 2003. After he received his Master he was appointed as a Lecturer at Faculty of Electrical Engineering. In 2005 he was appointed as a senior lecturer and in 2007 he was appointed as Assoc Professor at the faculty. Now he is the Professor in RF and Antenna at Faculty of Electrical Engineering Universiti Teknologi Malaysia. His research interest includes the areas of design of Dielectric resonator antennas, microstrip antennas, small antennas, microwave sensors, RFID antennas for readers and tags, Multi-function antennas, microwave circuits, EBG, artificial magnetic conductors, metamaterials, phased array antennas, computer aided design for antennas and design of millimeter frequency antennas. He has published over 200 articles in journals, conference papers, and book chapters.

Noor Asniza Murad holds a $\mathrm{PhD}$ in Electrical Engineering from The University of Birmingham (2011), Meng and BEng in Electrical Engineering from Universiti Teknologi Malaysia (UTM) in 2001 and 2003 respectively. She is a registered member of Board of Engineer Malaysia (BEM), The Institute of Electrical and Electronic Engineering (IEEE), and The Institution of Engineering and Technology (IET). She has one-year experience at HID GLOBAL Sdn. Bhd. on the evelopment of RFID tags antenna. She served the Universiti Teknologi Malaysia since 2001 starting as a tutor under the Department of Radio Communication Engineering. She is now a senior lecturer at Faculty of Engineering, Universiti Teknologi Malaysia. Her research interest mainly on antenna and sub-system design, RFID, antenna beamforming, as well as other RF components such as couplers, not only on planar devices but also waveguide based structures.

Farid Zubir (M'10) received the B.Eng. degree in Electrical majoring in Telecommunication as well as M.Eng. (RF \& Microwave) degree from the Universiti Teknologi Malaysia (UTM) in 2008 and 2010 respectively. In 2015, he then obtained his Ph.D degree from the University of Birmingham, UK, for research into direct integration of power amplifiers with antennas in microwave transmitters. He currently serves as a Senior Lecturer in the Department of Communication Engineering, School of Electrical Engineering, UTM. He is currently serves as a Postdoctoral Fellow at the University of British Columbia, Okanagan. His current research interest and specialization are in the area of RF and microwave technologies including planar array antenna, dielectric resonator antenna (DRA), active integrated antenna (AIA), microstrip reflectarray antenna (MRA), millimetre wave antenna, electromagnetic band gap (EBG), artificial magnetic conductor (AMC), metamaterial and microwave amplifier. He has published over 40 articles in journals, book chapters, conference papers and proceedings.

Huda A Majid received the B Eng. degree in Electrical Engineering (Telecommunication from Universiti Teknologi Malaysia, in 2007. He then obtained his M.Eng in 2010 and PhD degrees in Electrical Engineering in 2013, at Universiti Teknologi Malaysia. He is currently a lecturer in the Department of Electrical Engineering Technology, Faculty of Engineering Technology, Universiti Tun Hussein Onn Malaysia. His research interest includes the areas of design of microstrip antennas, small antennas, Reconfigurable antennas, metamaterials structure, metalaterial antennas and millimeter wave antennas. He has published over 100 articles in journals and conference papers. 